\title{
TEM Characterization of 3D InAs QDs Grown under Subcritical Deposition
}

\author{
V. Shchukin ${ }^{1}$, N. Ledentsov ${ }^{1}$ and S. Rouvimov ${ }^{2}$ \\ 1. VI Systems GmbH, Hardenbergstr. 7, Berlin D-10623, Germany, and \\ A. F. Ioffe Physical Technical Institute, Politekhnicheskaya 26, St. Petersburg 194021, Russia \\ 2. University of Notre Dame, 112 North Notre Dame Avenue, South Bend, IN 46556, USA.
}

Here we present several strategies for TEM/STEM analysis of InAs quantum dots (QDs) formed in GaAs matrix under subcritical deposition conditions in Stranski-Krastanow growth [1]. While typical QD formation in such growth mode usually includes two materials, A and B (e.g. GaAs and InAs) where B lattice-mismatched to substrate A, the new approach involves a third material, C (e.g. AlAs), that allows for a better control of size and distribution of QDs. It was shown that introducing the third material significantly affects the surface morphology [2] and leads to the formation of QD structures with a thinner wetting layer and a reduced accumulated strain. FEI-Titan 80-300 electron microscope operated at $300 \mathrm{kV}$ was employed for evaluation of QD structure and chemical composition in both TEM and STEM modes. TEM analysis provided an evidence for a new growth mechanism for QD formation below critical thickness in InAs/AlAs/GaAs QD structures.

Based on the experimental data, we propose the deposition of B below the critical thickness (Fig. 1), where a wetting layer forms but no 3D islands form yet, followed by the subsequent deposition of material $\mathrm{C}$ that does not wet $\mathrm{B}$ and has a small or zero mismatch to A. Theory addressing close-tosurface equilibrium growth conditions [3] is developed for a three-materials system. The earlier model [4] accounting elastic strain relaxation due to island formation, strain-induced renormalization of the surface energy, relaxation at the island edges, and elastic repulsion between islands is further extended including $\mathrm{B} / \mathrm{A}$ wetting- and $\mathrm{C} / \mathrm{B}$ non-wetting conditions and the instability of a $\mathrm{B}-\mathrm{C}$ surface alloy against phase separation. The model reveals surface structures containing $\mathrm{B}$-rich domains and $\mathrm{C}-$ rich domains of the wetting layer and the onset of 3D B-rich islands at an amount of the deposited B smaller than the critical amount in the reference B/A system.

Experimental proof has been given for a subcritical deposition of 1.5 monolayers (ML) InAs on GaAs followed by the deposition of $0.5 \mathrm{ML}$ AlAs. Cross-sectional scanning transmission electron microscopy (STEM) images taken at chemically sensitive conditions reveal the formation of AlAs-rich domains in the wetting layers (Fig. 2), in agreement with the model. Photoluminescence spectroscopy (PL) shows a $\sim 185 \mathrm{meV}$ red shift of the PL peak upon the deposition of AlAs (Fig. 3), related to the formation of Inrich 3D islands (QDs).

The advantages of the new growth mechanism includes less accumulated strain in the wetting layer hindering the formation of structural defects and allowing defect-free active medium for optoelectronic devices for a broader spectral range. This is particularly important for stacked QD structures. The phenomenon is expected to be universal and applicable also to other materials systems like $\mathrm{GaN} / \mathrm{InN} / \mathrm{AlN}$, etc.

References:

[1] Bimberg D, Grundmann M, Ledentsov N, Quantum Dot Heterostructures, Chichester: Wiley, 1998, 
[2] R. Bergamaschini, et al., Phys. Rev. Lett. 109, 156101 (2012).

[3] N. Ledentsov, et al. Semicond. Sci. Technol. 16, 502 (2001).

[4] V. Shchukin, et al. Phys. Rev. Lett. 75, 2968 (1995).

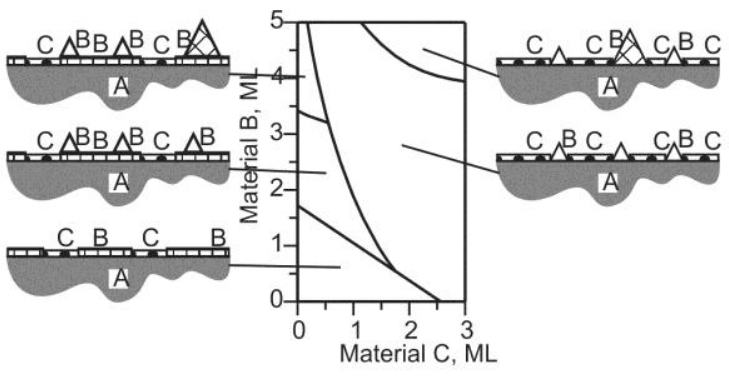

Fig. 1. Calculated phase diagram of the 3-materials C/B/A system. Left panel: Phases include a wetting layer consisting of mixed domains of $B$ and $C$ and (from bottom to top) no 3D islands, coherent $3 \mathrm{D}$ islands of $\mathrm{B}$, both coherent and dislocated $3 \mathrm{D}$ islands of B. Right panel: Phases include a wetting layer of C and (from bottom to top) coherent 3D islands of B, both coherent and dislocated islands of $\mathrm{B}$.
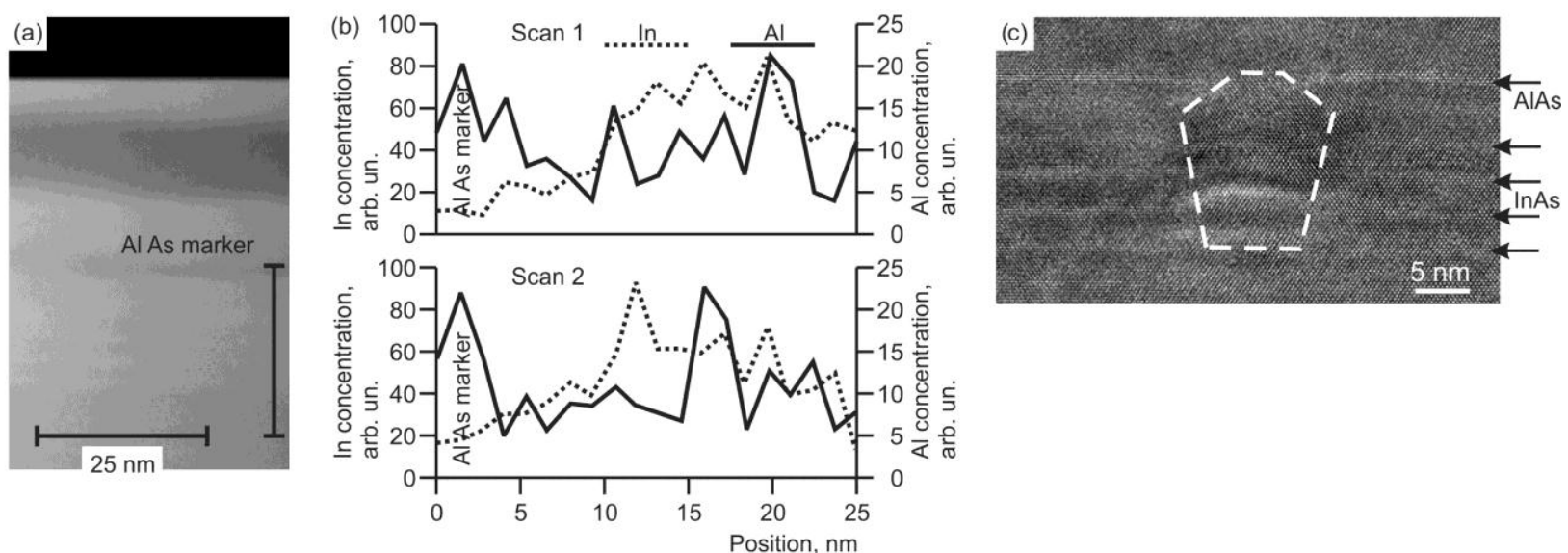

Fig. 2. (a) Cross-sectional scanning transmission electron microscopy image of 4 -fold stack of $A \mid A s / \ln A s / G a A s$ structures taken at chemically sensitive conditions. Bright contrast is In, dark contrast is Al, a thick layer on top is an AlAs marker.

(b) Two vertical scans taken at different positions show that, for each layer of deposited InAs/AIAs,

the concentration of Al drastically varies from scan to scan, which confirms the formation of Al-rich and Al-poor domains.

(c) Cross-sectional high resolution transmission electron microscopy image of a 4-fold stacked island.
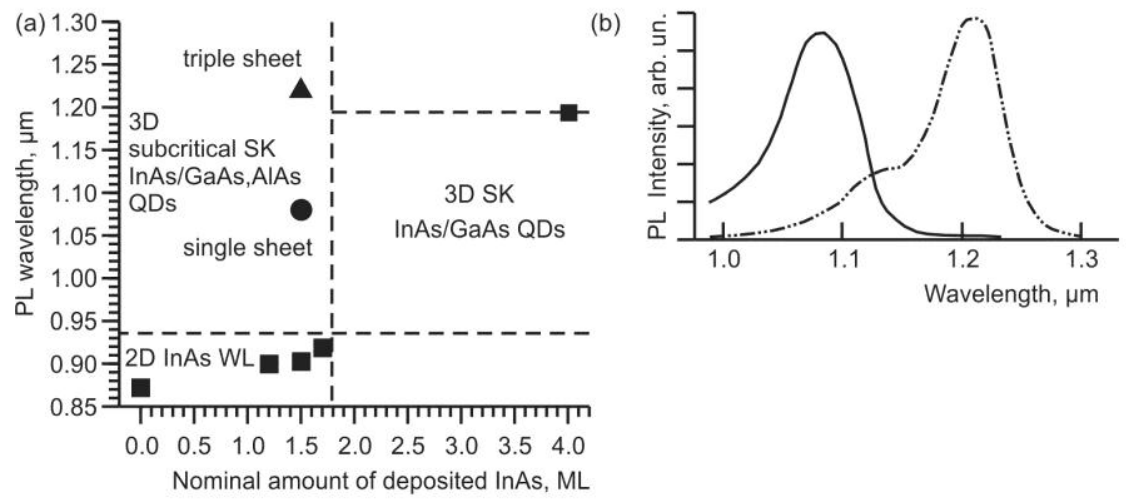

Fig. 3. (a) Spectral position of the photoluminescence (PL) peak in InAs/GaAs system with and without deposition of $0.5 \mathrm{ML}$ of AlAs. Deposition of AlAs on subcritical $1.5 \mathrm{ML}$ InAs/GaAs results in a significant red shift of the PL peak. (b) PL spectra at room temperature $\left(\sim 1 \mathrm{~W} / \mathrm{cm}^{2}, 532 \mathrm{~nm}\right)$ of the samples with a single sheet of InAs (solid line) and triple-stacked InAs with $2.5 \mathrm{~nm}$ GaAs spacers (dash-dotted line). 